\title{
Reconstruction of Hemipelvectomy Defect Using a Fillet Flap with Femoral Periosteum
}

\author{
Masaki Yazawa ${ }^{1}$, Tsuyoshi Kaneko ${ }^{2}$, Sumitoshi Katsumata ${ }^{3}$, Kazuo Kishi ${ }^{1}$ \\ ${ }^{1}$ Department of Plastic and Reconstructive Surgery, Keio University School of Medicine, Tokyo, Japan \\ ${ }^{2}$ Department of Plastic and Reconstructive Surgery, National Center of Child Health and Development, Tokyo, Japan \\ ${ }^{3}$ Department of Plastic Surgery, Chiba-Nishi General Hospital, Chiba, Japan \\ E-mail:yazawa@sc.itc.keio.ac.jp \\ Received August 6, 2010; revised August 6, 2010; accepted August 6, 2010
}

\begin{abstract}
Backgrounds: Reconstruction after hemipelvectomy is very important for rehabilitation into society. The pelvis plays an important role for support of the intra-abdominal organs. Methods: We operated 3 cases using fillet flaps with the femoral periosteum for reconstruction of hemipelvectomy defect. Results: It is useful to elevate the flap all around with the femoral periosteum, because the periosteum can be sutured to supporting pelvic structures with the aim to support intra-abdominal organs. Conclusion: Without alternative supports for the bony pelvis, pelvic reconstructions are at risk for hernia and it may be difficult for outpatients to fit their habiliments after radical cure. The rigid support for the intra-abdominal organs occurs in association with the suture pelvic and femoral periosteum.
\end{abstract}

Keywords: Hemipelvectomy, Reconstruction, Fillet Flap, Periosteum

\section{Introduction}

Hemipelvectomy is indicated for cases of primary pelvic or hip tumor without metastasis that are resectable for radical correction. Metastatic pelvic tumor that can be controlled at the primary focus, for example, a pelvic metastasis of thyroid cancer, is also indicated. Reconstruction after hemipelvectomy is important for the improvement of quality of life in the early postoperative stage and for rehabilitation into society. In the reconstruction after hemipelvectomy, some cases require revascularization and urinary diversion, however, plastic surgeons are needed for wound closure for skin defects and filling dead spaces using flaps [1,2]. In cases of hemipelvectomy, some innovative techniques for skin defects have been reported. Most of them have used local thigh flaps without tumor invasion for pelvic closure [3-6]. The pelvis plays an important role for support of the intra-abdominal organs. Without alternative supports for the bony pelvis, pelvic reconstructions are at risk for hernia and it may be difficult for outpatients to fit their habiliments after radical cure. Some cases that have the benefit of using the lateral thigh can usually obtain enough support using the strong tensor fascia lata. We report cases using fillet flaps with the femoral perio- steum for reconstruction of hemipelvectomy defect.

\section{Case Report}

\subsection{Case 1}

A 42-year-old man underwent resection of an osteosarcoma in the right greater trochanter. A giant tumor was found around the hip joint, and it was necessary to extensively remove skin (Figure 1). The tumor was completely removed and a safety margin was kept. The flap was planned at the medial thigh with femoral periosteum, and the vascular pedicle was external iliac vessels. But this flap was not planned as a complete island flap because we wanted to preserve the sensory of skin near the ischium (Figures 2, 3). This femoral periosteum was sutured to the pelvic periosteum (Figure 4), and dead spaces in the pelvis were filled with the muscle in the flaps and the pelvic stump was covered with the skin portions of the flap. No recurrence has been seen 8 months postoperatively (Figure 5). Wearing an artificial limb, he can walk with the help of crutch.

\subsection{Case 2}

A 15-year-old woman underwent resection of a chon- 


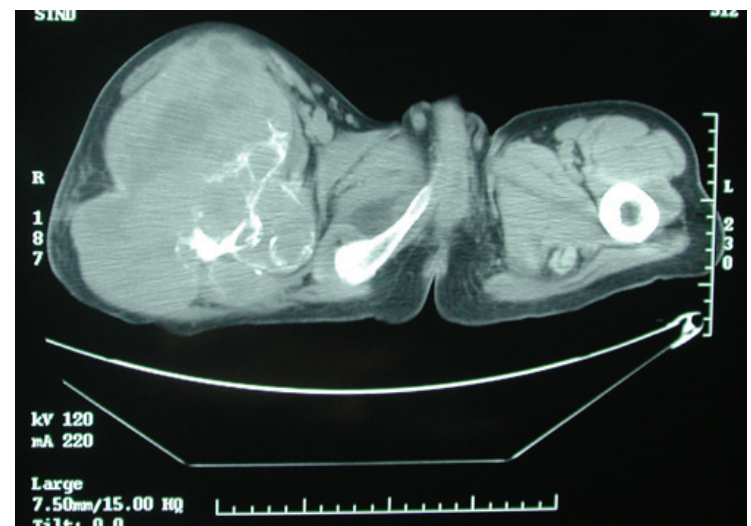

Figure 1. Preoperative CT showing an osteosarcoma in the right greater trochanter.

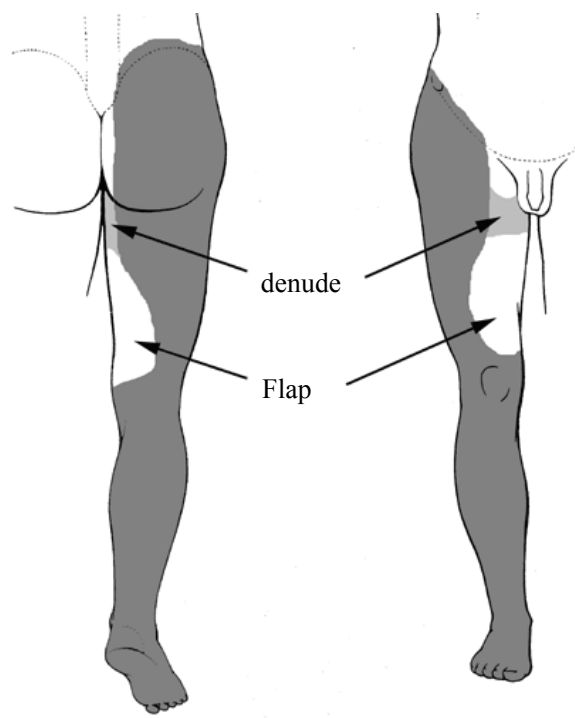

Figure 2. Schematic illustration of the operation. Excised tissue is in dark gray. Denuded area to preserve the sensory of skin near ischium is in light gray.

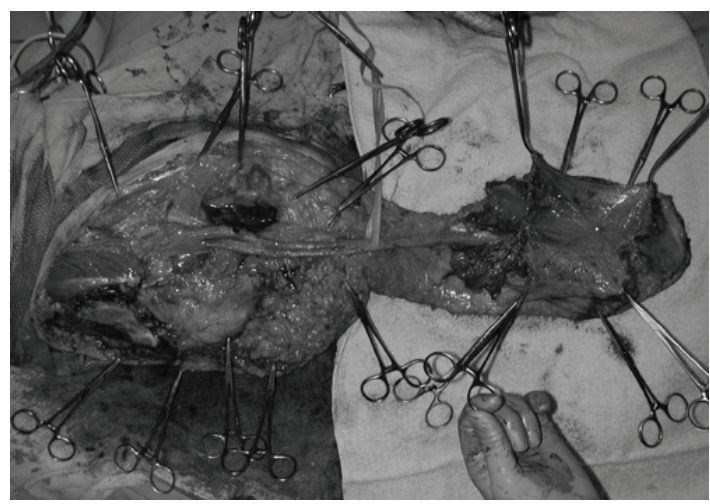

Figure 3. The elevated medial thigh flap was connected with vascular pedicle to the trunk. The pedicle is external iliac vessels divided into common femoral vessels. The center of this flap is the femoral periosteum.

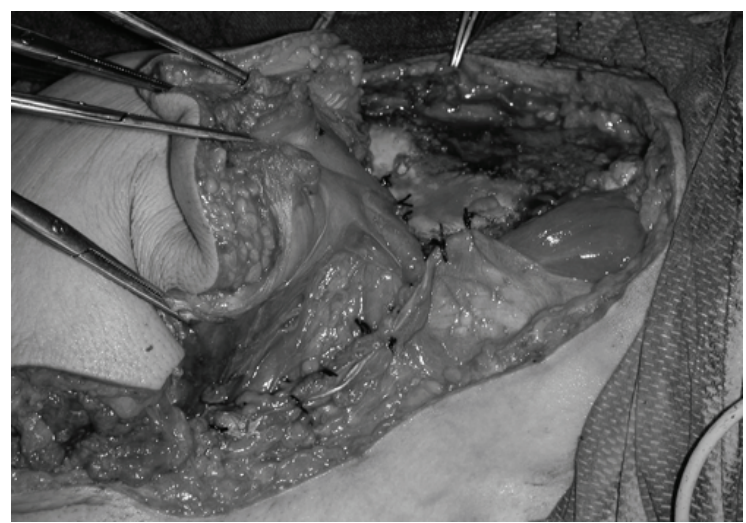

Figure 4. Femoral periosteum sutured to the sacral periosteum posterior to the bone.

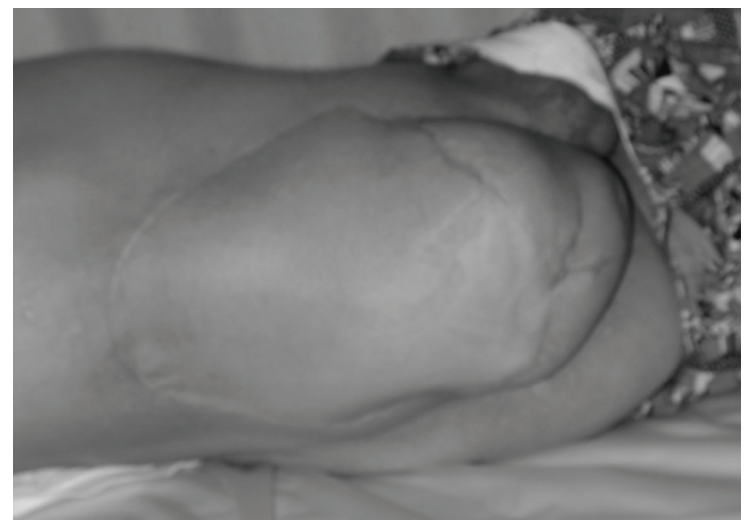

Figure 5. Eight-month postoperative lateral view.

drosarcoma in the left pelvis. Strong radiodermatitis had occurred in the gluteal and posterior thigh region by preoperative radiation therapy, so we decided to use a musculocutaneous flap with periosteum from the medial thigh for pelvic reconstruction (Figure 6). Following hemipelvectomy, the tumor was completely removed and a safety margin was kept. The flap was planned at the medial thigh as an island flap with femoral periosteum, and the vascular pedicle was external iliac vessels. As with Case 1, this femoral periosteum was sutured to the pelvic periosteum, and dead spaces in the pelvis were filled with the muscle in the flaps and the pelvic stump was covered with the skin portions of the flap. No recurrence has been seen 4 years postoperatively.

\subsection{Case 3}

A 65-year-old man underwent resection of metastatic bone tumors from thyroid cancer in the left iliac bone. He had already received radiotherapy with a total amount of $60 \mathrm{~Gy}$. The tumor was completely removed and a safety margin was kept. The flap was planned at the anterior thigh with femoral periosteum, and the vascular 


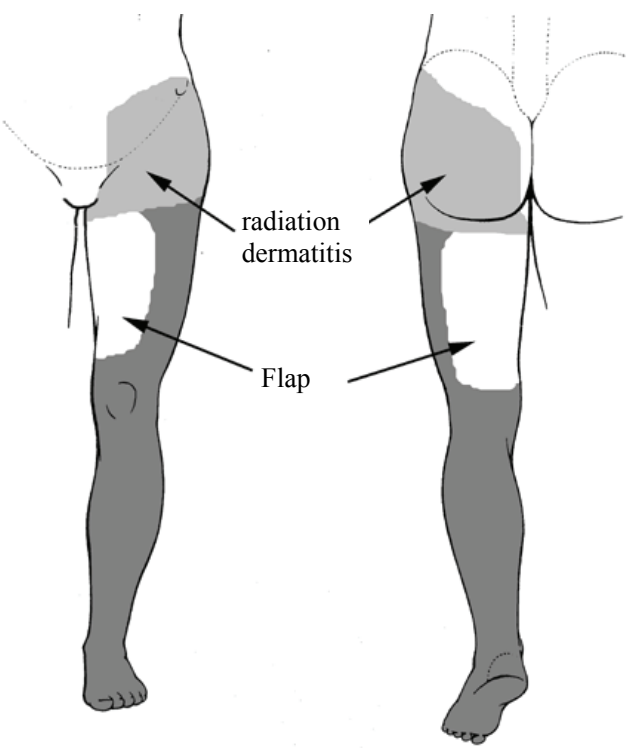

Figure 6. Schematic illustration of the operation. Excised tissue is in dark gray. Radiation dermatitis is in light gray.

pedicle was external iliac vessels. As with Case 1, this flap was not planned as a complete island flap, because we wanted to preserve the sensory of skin near the ischium (Figure 7). As with Cases 1 and 2, the femoral periosteum was sutured to the pelvic periosteum, and the dead spaces in the pelvis were filled with the muscles in the flap and the pelvic stump was covered with skin portions of the flap. This patient could get around in a wheelchair 2 weeks after the operation, and he could maintain a standing position between parallel bars 3 weeks after the operation.

\section{Discussion}

Reconstruction after hemipelvectomy by plastic surgery is very important for rehabilitation into society. In the hemipelvectomy, pelvic tissue and various lower limbs are removed depending on the stages of cancer progress. Simple reconstruction for the skin defect and dead spaces in the pelvis gives birth to complications. Especially in the hemipelvectomy, various complications, for example, infection, poor adaptation, pelvic dead space infection, ileus, and hernia, are frequently reported. Poor suturing, excessive weight of intra-abdominal organs against wounds, and the existence of pelvic dead spaces may cause these complications. These complications not only prolong wound repair but also disturb postoperative radiotherapy, chemotherapy, and rehabilitation into society. In our procedure, it is important to have a conversation preoperatively with orthopaedists about the amount of removal, whether large vessels will be involved (external iliac artery and vein, etc.), operative body position, past

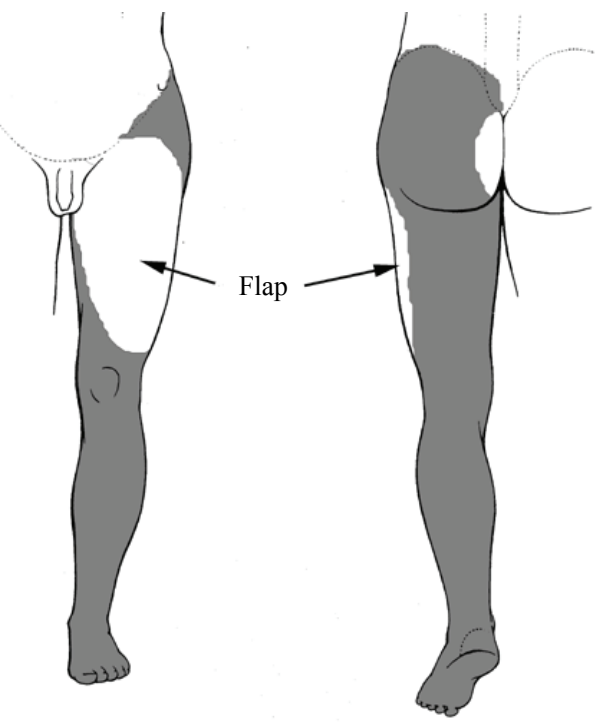

Figure 7. Schematic illustration of the operation. Excised tissue is in dark gray.

history (radiation therapy, etc.), scheduled postoperative therapy and expected activities of daily living. Musculocutaneous flaps from the gluteal region and posterior thigh are usually available. If they are not available because of tumor invasion and the influence of radiation therapy, a local flap from the medial or anterior thigh can be used. Flaps with external iliac vessels or femoral vessels are planned distally from tumors and are elevated as musculocutaneous flaps with femoral periosteum. The blood supply to this periosteum is from the deep femoral artery given off by the femoral artery. The rigid support for the intra-abdominal organs occurs in association with the suture femoral and pelvic periosteum from the pubis to the sacral. The femoral periosteum can be sutured not only to the pelvic periosteum anterior or posterior to the bone but also to the pelvic bone itself. Muscles in the flaps play the role of filler to dead spaces and a cushion against habiliments. Because of the progress in plastic surgery involving blood circulation, the volume of flaps for large skin defects, and pelvic dead spaces, these problems can be solved in most cases. In addition, wider tumor radical surgery became possible in several types of surgeries [7], including hemipelvectomy. However in hemipelvectomy, this technique sometimes leaves flaps that are unable to hold up the intra-abdominal organs.

It is difficult in hemipelvectomy to keep a clean surgical field because of the long operation time. So, in addition to the preoperative radiotherapy, postoperative infection odds are high. Accordingly, the tissue for the closure of the pelvic space must have good blood circulation. In cases of poor support after the reconstruction, we sometimes use not only artificial materials like proline mesh but also free nonvascularized fascia. In several 
cases, the tumor, even in the indication for the hemipelvectomy, rarely extends to the lower limb. If the flap from the lateral or posterior thigh is available, tensor fascia lata and gluteal muscles are useful. If, however, only the flap from the anterior or medial thigh is available, enough support may not be given to the reconstruction, because the flaps from the anterior and medial thigh are weaker than the flaps from the lateral and posterior thigh. Therefore, the anterior and medial thigh flaps may result in postoperative displacements of intra-abdominal organs. Consequently, it is useful to elevate the flap all around with the femoral periosteum, because the periosteum can be sutured to supporting pelvic structures with the aim to support intra-abdominal organs. Periosteum is widely available in the thigh flaps without tumor invasion to the femur. In Cases 1 and 2, the covering skin in the flap was not sufficient, and it was necessary to elevate the free flap from the distal limb after the amputation and to inosculate the vessels at the pelvic stump $[8,9]$. A free flap requires time-consuming operation and is at risk for vascular anastomosis. On the other hand, by preserving femoral vessels, it is possible to elevate the musculocutaneous flap with femoral periosteum and to use vascularized pedicle without vascular anastomosis. This fillet flap with periosteum is a useful choice for reconstruction after hemipelvectomy and extends the possibilities for the indication of hemipelvectomy.

\section{References}

[1] W. H. O. Bohne, "Atras of Amputation Surgery, Hemipelvectomy," New York Thieme Medical Publishers, Inc., New York, 1987.
[2] M. W. Chapman, "Operative Orthopaedics, Amputations," Philadelphia J. B. Lippincott Company, Philadelphia, 1993.

[3] D. W. Chang, J. E. Lee, Z. L. Gokaslan and G. L. Robb, "Closure of Hemicorporectomy with Bilateral Subtotal Thigh Flaps," Plastic and Reconstructive Surgery, Vol. 105, No. 5, 2000, pp. 1742-1746.

[4] C. E. Butler, "Reconstruction of an Extensive Hemipelvectomy Defect Using a Pedicled Upper and Lower Leg in-Continuity Fillet Flap," Plastic and Reconstructive Surgery, Vol. 109, No. 3, 2002, pp. 1060-1065.

[5] N. B. Meland, E. J. Ivy and J. E. Woods, "Coverage of Chest Wall and Pelvic Defects with the external Oblique Musculofasciocutaneous Flap," Annals of Plastic Surgery, Vol. 21, No. 4, 1988, pp. 297-302.

[6] D. L. Larson and M. D. Liang, "The Quadriceps Musculocutaneus Flap: A Reliable, Sensate Flap for the Hemipelvectomy Defect," Plastic and Reconstructive Surgery, Vol. 72, No. 3, 1983, pp. 347-353.

[7] M. V. Kuntscher, D. Erdmann, H. H. Homann, H. U. Steinau, S. L. Levin and G. Germann, "The Concept of Fillet Flaps: Classification, Indications, and Analysis of their Clinical Value," Plastic and Reconstructive Surgery, Vol. 108, No. 4, 2001, pp. 885-896.

[8] M. L. Workman, D. F. Bailey and B. L. Cunningham, "Popliteal-Based Filleted Lower Leg Musculocutaneous Free-Flap Coverage of a Hemipelvectomy Defect," Plastic and Reconstructive Surgery, Vol. 89, No. 2, 1992, pp. 326-329.

[9] Y. Yamamoto, H. Minakawa and N. Takeda, "Pelvic Reconstruction with a Free Fillet Lower Leg Flap," Plastic and Reconstructive Surgery, Vol. 99, No. 5, 1997, pp. 1439-1441. 